\title{
Circulating tumor-cell detection and capture using microfluidic devices
}

\author{
Q2 Laszlo Hajba a , Andras Guttman a,b,* \\ a MTA-PE Translational Glycomics, MUKKI, University of Pannonia, Veszprem, Hungary \\ b Horvath Laboratory of Bioseparation Sciences, University of Debrecen, Debrecen, Hungary
}

\section{A R T I C L E I N F O}

\section{Keywords:}

Cancer-therapy management

Circulating tumor cell (CTC)

CTC capture

CTC detection

Dielectrophoresis

Early detection

Hydrodynamics

Metastases

Microfluidics

Nanotechnology

\begin{abstract}
A B S T R A C T
Circulating tumor cells (CTCs) in the bloodstream are considered good indicators of the presence of a primary tumor or even metastases. CTC capture has great importance in early detection of cancer, especially in identifying novel therapeutic routes for cancer patients by finding personalized druggable targets for the pharmaceutical industry. Recent developments in microfluidics and nanotechnology improved the capabilities of CTC detection and capture, including purity, selectivity and throughput. This article covers the recent technological improvements in microfluidics-based CTC-capture methods utilizing the physical and biochemical properties of CTCs. We critically review the most promising hydrodynamic, dielectrophoretic and magnetic force-based microfluidic CTC-capture devices.
\end{abstract}

(C) 2014 Elsevier B.V. All rights reserved.

\section{Contents}

1. Introduction

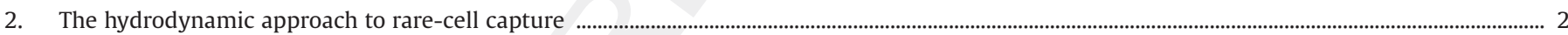

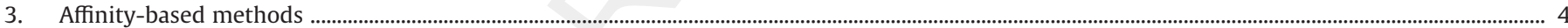

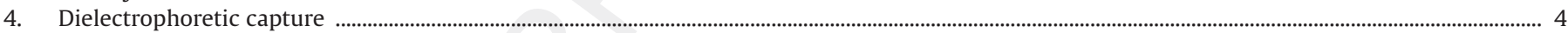

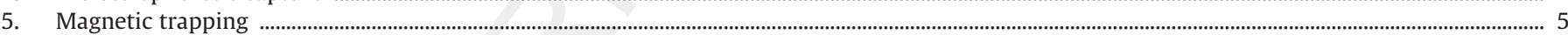

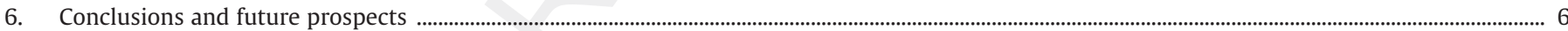

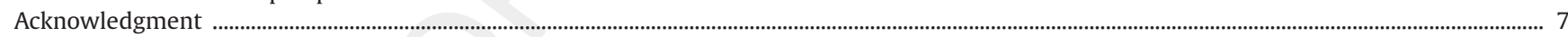

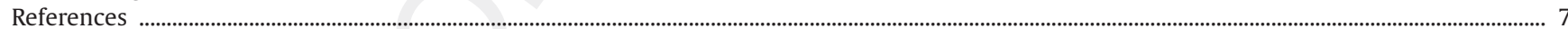

\section{Introduction}

Tumor cells can shed into the bloodstream at a very early stage of primary tumor growth and these so called circulating tumor cells (CTCs) may give rise to metastases at distant parts of the body [1].

Abbreviations: BM, Bone marrow; CTC, Circulating tumor cell; DEP, Dielectrophoresis; EGFR, Epithelial growth factor receptor; EpCAM, Epithelial cell adhesion molecule; HeLa, Cervical cancer cell line; HER, Human epidermal growth factor receptor; LNCaP, Androgen-sensitive human-prostate adenocarcinoma cell; mAb, Monoclonal antibody; MCF-7, Michigan Cancer Foundation-7 breast-cancer cell line; MDA-MB-231, Breast-cancer cell line; NSCLC, Non-small cell lung cancer; OECM1, Human oral squamous cancer cell line; PBS, Phosphate buffered saline; PC-3, Human prostate-cancer cell line; SCLC, Small-cell lung cancer; SKOV-3, Ovarian cancer cell line; SW-620, Colon adenocarcinoma cell line.

Q1 * Corresponding author.

E-mail address: a.guttman@neu.edu (A. Guttman)
Detecting the presence of viable CTCs therefore provides a good indicator for the presence of malignant transformations [2]. Isolation and analysis of CTCs is important to improve understanding of their nature, and their ability to initiate metastases, and for effective development of next-generation cancer therapeutics [3-5].

Primary tumors are usually detected at the stage of clinical symptoms by the non-invasive means of imaging techniques, such as traditional radiography (X-ray), magnetic resonance imaging (MRI), computed tomography (CT), and ultrasound, or with invasive biopsy collection. For example, cells shed from a primary tumor can accumulate in the bone marrow (BM) [6], necessitating invasive BM biopsy to see their presence and possibly to monitor the effect of a therapy. CTC-detection technologies can help substitute an invasive biopsy for a simple blood draw, also referred to as liquid biopsy [7-9].

At present, CTC-detection methods are not routinely used by clinicians to make therapeutic decisions, mostly because of the lack 
of randomized data showing that therapies based on the CTC numbers show improvement in prognosis [7]. The analytical validity of CTC-detection systems is crucial for their clinical acceptance, as emphasized in the guidelines recently published by the US Food and Drug Administration (FDA) [10]. As of today, the only FDA-approved CTC-detection method is the CellSearch System [11], which is based on immunomagnetic detection of EpCAM positive cells.

The most challenging part of effective CTC analysis is the cellcapture design because of the extremely low abundance or rareevent nature of these cells. For example, it is of great clinical importance to enumerate quantitatively $0-10$ CTCs per $\mathrm{mL}$ in whole blood that also contains $>10^{9}$ erythrocytes and $>10^{6}$ leukocytes [12]. For sampling rare events in a large cell population, the following should be considered:

(1) throughput, i.e. the number of cell identification or sorting steps per unit time;

(2) recovery, in the sense of the number of target cells collected from the input sample; and,

(3) purity, which depends on the number of "interfering" cells excluded from the analysis [13].

Microfluidic systems with small sample-volume requirement, fast processing times, multiplexing capabilities and large surface areato-volume ratios offer an option for in-vitro cell sorting, detection and single-cell analysis [14]. Based on these advantages, various microfluidic platforms have been developed for rare-cell capture, including CTCs [15]. Recent progress in microbiotechnology and nanobiotechnology can also aid the development of more advanced CTC-detecting microfluidic devices [16].

Molecular profiling of CTCs can provide valuable information about genetic mutations and can identify therapeutic targets for personalized treatment [17-19]. For example, in primary breast cancer, human epidermal growth factor receptor-2 (HER2) is an important treatment target, so presence of HER2-positive CTCs could be a good indication for anti-HER2 treatments. Even in case of a HER2negative primary tumor verified by tissue biopsy, it might be reasonable to use anti-HER2 treatment if HER2-positive CTCs are found. Independent of the HER2 status of the primary tumor, the number of HER2-positive CTCs usually correlates with decreased diseasefree survival and decreased overall survival [20]. Large prospective clinical trials have already been running to prove the efficiency of anti-HER2 therapy based on the presence of HER2-positive CTCS [21]. Another therapeutic target is the epithelial growth factor receptor (EGFR) in non-small cell lung cancer (NSCLC) patients. Maheswaran et al. identified a mutation in EGFR by genotyping of CTCs that cause drug resistance in NSCLC patients against EGFRinhibitor drugs [22]. Monitoring the tumor genotypes during lungcancer patient treatment, CTCs may provide information about possible acquired resistance against EGFR-inhibitor drugs, so as to help clinicians in therapeutic decision making.

In this article, we review the new advances and the state of the art of the most commonly used CTC-capture methods for downstream analysis utilizing hydrodynamic, dielectrophoretic and magnetic forces to provide an adequate number of these important marker cells for use in biomedical and clinical applications and development of next-generation therapeutics.

\section{The hydrodynamic approach to rare-cell capture}

Hydrodynamic cell-capture methods in microfluidic settings greatly depend on the interaction between the species of interest and the microfluidic channel walls, and the sample-fluid matrix. In this section, we discuss label-free hydrodynamic capture methods that are based on size, shape and cell deformability. The cell-wall interaction can be further divided into continuous wall (channels) and discrete wall (obstacles) [23]. In a continuous wall, the microfluidic channel has a flat surface and the flow fractionation of cells is controlled by pressure [24] or tuned by electroosmotic flow [25]. In the discrete wall, the obstacles are usually posts and/or ridges. Through a periodic array of $\mu \mathrm{m}$-scale posts, with each row of posts shifted horizontally with respect to the previous row, cells above a critical size cannot be carried by the net vertical flow but are laterally displaced at each row. This lateral displacement is also affected by the deformability of the cells. The critical size can be calculated from the geometrical parameters of the posts (i.e. diameter, gap between posts, and row shift) [26].

Based on the dominant forces, hydrodynamic separation can be further classified into laminar-based, inertia-based and biomimeticsbased flows [23].

In laminar-flow-based microfluidics where only the viscous force is dominant $(\operatorname{Re}<1)$, a cell follows a path that goes through its center of mass. With carefully-designed flow in a pinched segment of the microchannel, the cells can be positioned in such a way that the cells of different sizes follow different paths and are consequently separated by size [27]. At higher Reynolds number $(1<\operatorname{Re}<100)$, the inertial force becomes the dominant separation force [28].

Biomimetics-based microfluidic devices have biologically inspired structural designs that can mimic physiological processes or use biological principles (e.g. blood flow in microcirculation). A good example is a microfluidic device that was developed to separate blood cells using biomimetics [29]. The microchannels were designed so that they mimicked microcirculation and enhanced the lateral migration of leukocytes. The average diameter of leukocytes is in the range $10-12 \mu \mathrm{m}$ [29] and the diameter of erythrocytes is $6.2-8.2$ $\mu \mathrm{m}$ [30]. The red blood cells (erythrocytes) were concentrated in the center of the microchannel, while the white blood cells (leukocytes) laterally migrated to near the microchannel walls. The biomimetics-based microfluidic device can achieve a 34-fold enrichment of the leukocyte-to-erythrocyte ratio [29].

Microfiltration is another useful technique for size-based rarecell trapping. Membrane microfilters are designed with welldefined pores to restrict passage for cells above a critical size. Similar to other types of filtration methods, any particulates commensurable with the pore size can cause clogging. The usual size of a CTC is $>8 \mu \mathrm{m}$, which is greater than $90-95 \%$ of the largest blood-cell population, erythrocytes (red blood cells) [31,32]. Zheng et al. [33]. designed a membrane microfilter with $11-\mathrm{m}$ circular pores and tested it with a prostate-cancer cell line (LNCaP) diluted in human-blood samples from healthy donors. With the average size of LNCaP cells of $17 \pm 1.5 \mu \mathrm{m}$, the recovery rate of membrane filtration was $87.3 \pm 7.0 \%$. An improved 3D microfiltration approach was presented by the same authors with the goals of minimizing the stress on the cell membrane and sustaining cell viability with the same recovery rate [34].

Microcavity arrays represent another special technique for sizebased capture of CTCs, as shown in Fig. 1. The microcavity-based microfluidic device was tested with peripheral blood from healthy donors spiked with MCF-7 and SW-620 tumor cells. Microcavity sizes were varied in the range 8-11 $\mu \mathrm{m}$. The recovery rates for MCF-7 cells (diameter $22.5 \mu \mathrm{m}$ ) remained almost constant with different microcavity sizes at a level $>90 \%$. For the smaller tumor cells of SW620 (diameter $11.6 \mu \mathrm{m}$ ), the maximum recovery rate of $88 \%$ was obtained using the average microcavity diameter of $9.1 \mu \mathrm{m}$ [35]. A rectangular type of microcavity array was also developed for effective capture of SCLC cells using microcavities with 5-9 $\mu \mathrm{m}$ width and $30 \mu \mathrm{m}$ length [36]. For SCLC cells, the recovery rate was $\sim 80 \%$.

Hydrodynamic lifting was recently developed by Geislinger et al. for blood-cell separation [37]. The principle of the method was the utilization of non-inertial hydrodynamic cell-wall interaction [i.e. at very low Reynolds numbers $(\operatorname{Re}<1)$, no inertial effects occur and 


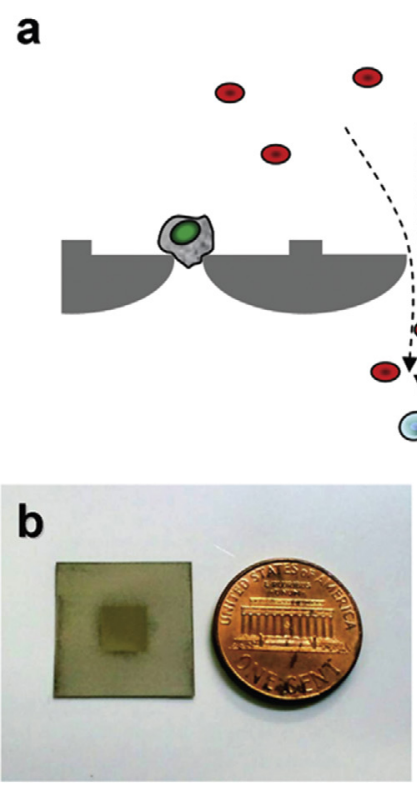

\section{d}

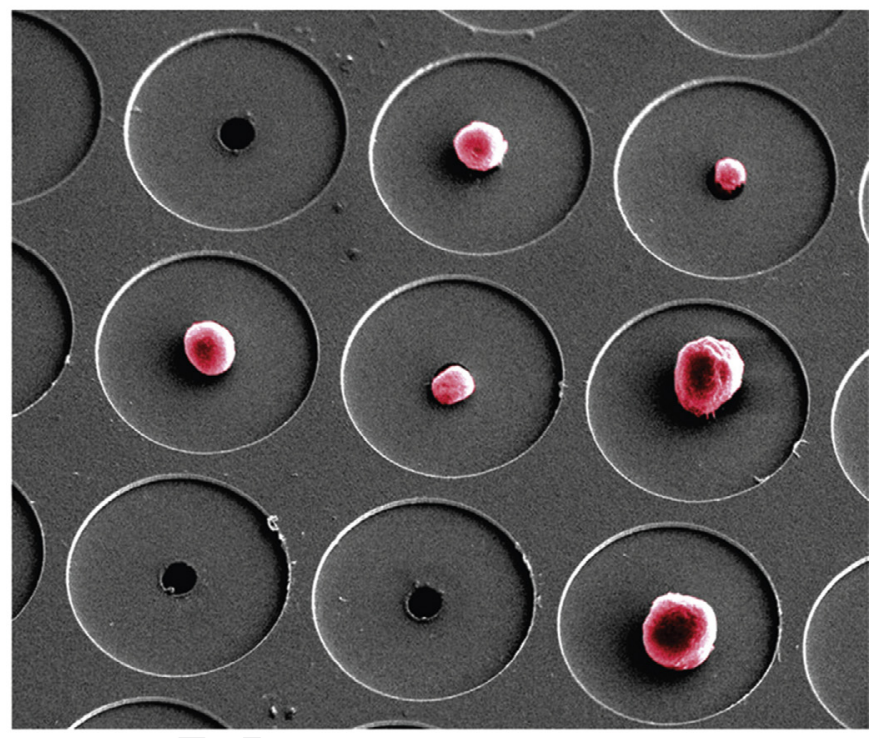

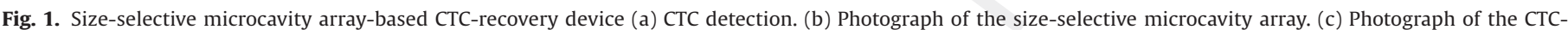

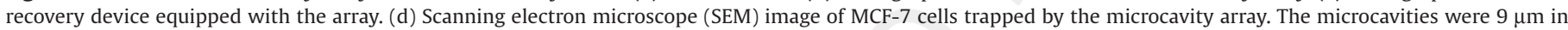
size, with a $60 \mu \mathrm{m}$ pitch. (@American Chemical Society [35]) A

the hydrodynamic lift (as a purely viscous effect) can provide adequate cell separation] [38]. As shown in Fig. 2, the cells in the sample flow enter through a smaller inlet channel to the chamber where the sample flow is hydrodynamically focused by a perpendicular sheath flow. The square cross-section of the sheath flow and sample inlets were $110 \mu \mathrm{m} \times 110 \mu \mathrm{m}$ and $110 \mu \mathrm{m} \times 30 \mu \mathrm{m}(\mathrm{y}$ - and $\mathrm{z}$ - directions), respectively. The main parameters that influenced the lateral lift velocity were cell size and deformability. The cell radius contributed cubically and cell deformability linearly to the lift velocity, respectively. This lab-on-a-chip system design could also separate CTCs from blood samples.

The selectivity of hydrodynamic lifting-based cell sorting was further enhanced by the application of spiral geometry. In this case, the dominant inertial force and a secondary rotational flow-field perpendicular to the original flow direction (Dean flow) cause the smaller particles to migrate in the direction of the outer half of the channel and the bigger particles migrate towards the inner channel wall $[39,40]$. This technique, referred to as Dean flow field fractionation, can be applied to separate CTCs from blood cells with high selectivity, based on their size differences [41]. Using double spiral geometry (Fig. 3), the separation efficiency at the inner outlet of the microchannel was found to be $98.72 \%$ for a binary test mixture of $5-\mu \mathrm{m}$ and $15-\mu \mathrm{m}$ diameter polystyrene particles [42]. After the promising test results, experiments were conducted with $20 \times$ diluted human blood drawn from healthy volunteers and spiked with HeLa cells (tumor-to-blood cell ratio $8 \times 10^{-7}$ ). At $60 \mathrm{~mL} / \mathrm{h}$ flow rate, the separation efficiency for HeLa cells was $90.546 \pm 3.41 \%$ with throughput of $2.5 \times 10^{8}$ cells $/ \mathrm{min}$. Please note that the separation efficiency was defined as the ratio of the number of one cell type collected at the desired outlet (the inner outlet for HeLa cells or the middle outlet for blood cells) to the total number of this kind of cell at all outlets.

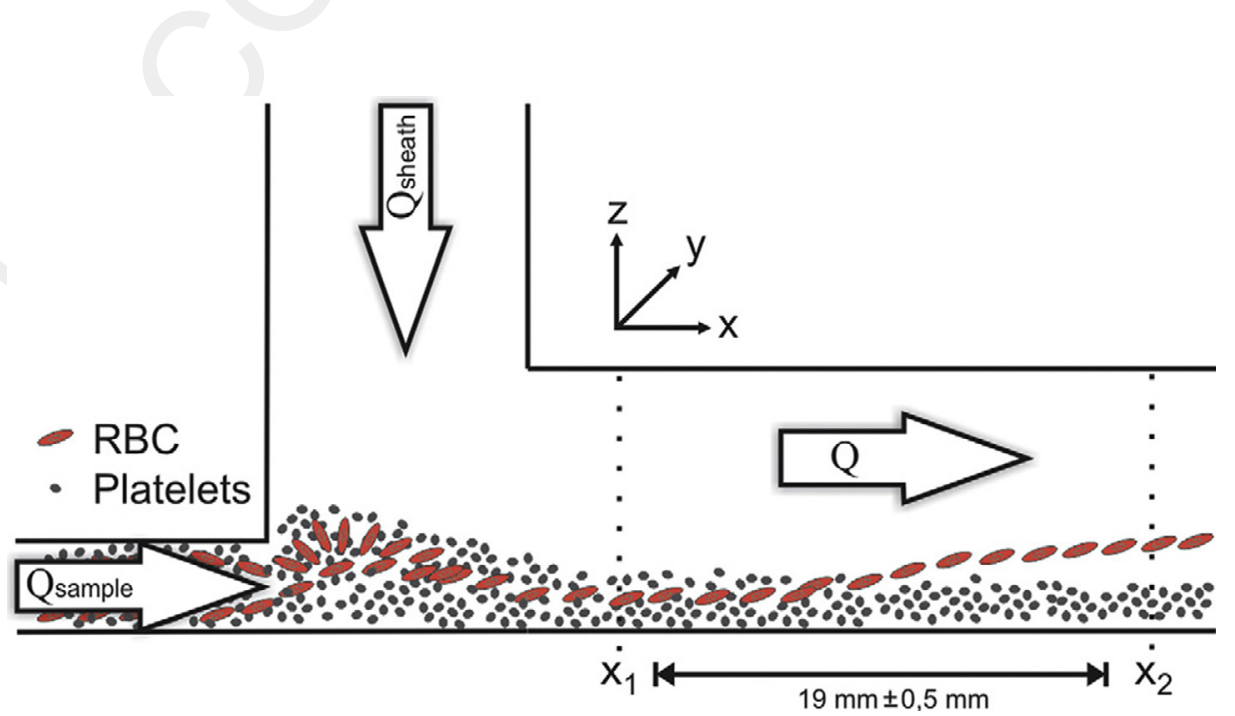

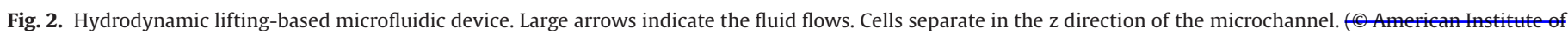
Physies $[37]$ ) 


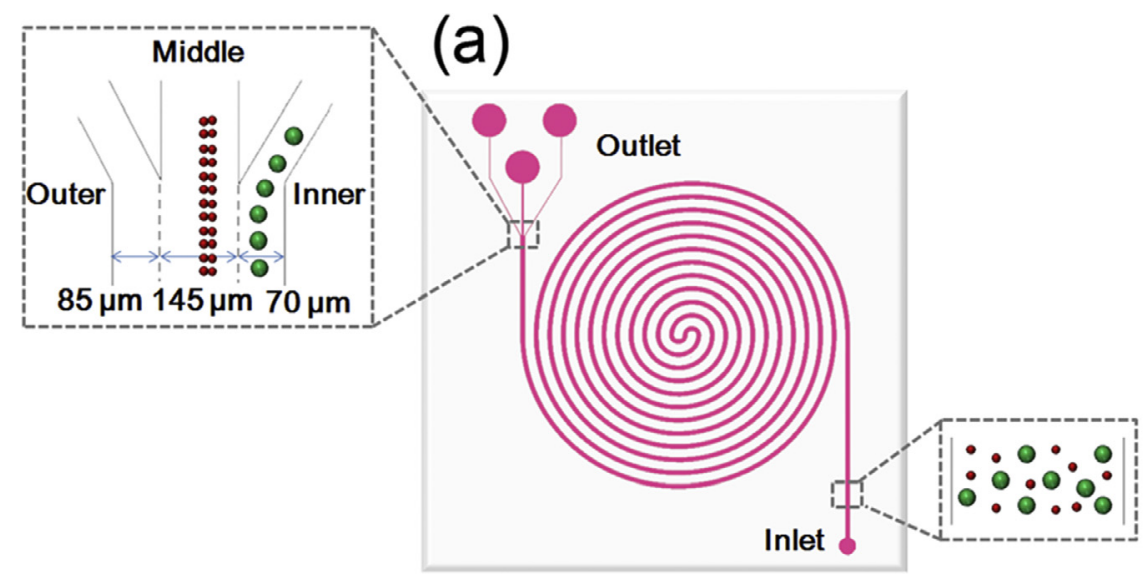

Fig. 3. (a) Microfluidic cell sorter containing a six-loop double-spiral microchannel. (b) Picture of the assembled cell sorter. (@-Ameriean

\section{Affinity-based methods}

Although recent developments in the field considerably increased the separation efficiency of hydrodynamic label-free methods, the maximum capture yield was still only around $90 \%$. To improve capture efficiency, special microposts were formed by photolithography in the channels of microfluidic devices and coated with CTC-specific antibodies, such as anti-EpCAM [43]. The two essential parameters that determined the efficiency of cell capture in an affinity-coated microfluidic device were:

(1) flow velocity, because it influenced the duration of cellmicropost contact; and,

(2) low shear force to ensure maximum cell-micropost attachment, which depends on the micropost distribution in the microchannel [44].

A geometrically-enhanced affinity-based microfluidic device reportedly captured $97 \pm 3 \%$ of spiked LNCaP cells from PBS [45]. The local topographical interaction can be enhanced by $3 \mathrm{D}$ nanostructured cell-capture substrates. Densely-packed nanopillars were prepared with diameters of 100-200 nm using wet chemical etching, followed by coating with the relevant antibody (antiEpCAM). Cell-capture experiments were performed by employing different nanopillar lengths in the range 4-20 $\mu \mathrm{m}$. It was reported that the capture yield for EpCAM-positive cells increased with nanopillar length [46]. Optimal capture yield was attained when the nanopillar lengths were comparable with the cell-protrusion lengths of the CTCs [47].

To enhance antibody-CTC interaction, a special hydrogel was developed. A degradable alginate biopolymer coating enabled gentle, efficient release of antibody-captured cells from the microfluidic device. The bottom of the microfluidic channel was coated with the antibody (anti-EpCAM)-functionalized alginate biopolymer. PC-3 cells were spiked into whole blood from a healthy donor and captured in the microfluidic device. After the capture, alginate lyase was pushed through the microfluidic channel, degrading the hydrogel (alginate biopolymer) and releasing the captured cells [48]. The advantage of this approach was that this hydrogel coating minimized non-specific interactions and enabled highly efficient release of the isolated cells $(99 \% \pm 1 \%)$ following hydrogel dissolution without any effect on cell viability.

A good example of the combination of hydrodynamic and affinitybased cell capture was published by Stott et al. [49]. Their herringbone-chip design enabled passive mixing of blood cells through the generation of microvortices that significantly increased the number of interactions between target CTCs and the antibody-coated chip surface. The herringbone chip was tested with the PC-3 prostate cancer cell line. The whole blood from healthy donors was spiked with PC-3 cells at a concentration of 500 cells $/ \mathrm{mL}$. The capture efficiency was $91.8 \pm 5.2 \%$ for the spiked PC-3 cells.

\section{Dielectrophoretic capture}

Dielectrophoresis (DEP) is a phenomenon by which a subject (e.g. cell) is forced to move by the application of divergent electric field gradients due to polarization forces. DEP forces depend on different factors, such as cell-membrane and cytoplasm-charge properties, and size. The electric field induces charges within the cell, forming dipoles. If the cell is more polarizable than the suspending medium, it is attracted towards the regions of higher electric fields, and the motion is called positive DEP. Conversely, if the cell is less polarizable than the suspending medium, it is repelled from the regions of higher electric field, and the motion is called negative DEP [50,51].

DEP techniques can also be distinguished by microelectrode type. Metallic microelectrodes with various geometries can be used \{e.g. interdigitated [52], castellated [53], oblique [54], spiral [55], circular [56], ring shape [57], and wedge shape [58], that are patterned on a microfluidic wafer using conventional lithography techniques\}.

Contactless DEP (cDEP) was developed for cell manipulation and sorting [59] so that the conventional metallic microelectrodes were replaced by fluidic electrode channels, filled with high-conductivity fluids. Application of this technique, eliminated problems (e.g. bubble formation and electrode-sample contamination) [60] and decreased fabrication costs.

Davis and co-workers recently developed a high-throughput continuous-flow DEP microfluidic device, named ApoStream [61], where an AC electric field was applied to the sample within optimized flow-path regions. The floor of the flow chamber comprised a flexible polyimide film sheet with electroplated copper and gold electrodes. An acrylic sheet formed the ceiling and a gasket the side walls. The sample was injected through a port located at the floor of the flow chamber at the same upstream end as the elution buffer. Cancer cells were collected through another port located downstream from the sample inlet port (Fig. 4).

With the setting shown in Fig. 4, the average recovery of SKOV-3 and MDA-MB-231 cancer cells, spiked into $12 \times 10^{6}$ peripheral blood mononuclear cells obtained from normal human-donor blood, was $75.4 \% \pm 3.1 \%$ (number of samples was 12 ) and $71.2 \% \pm 1.6 \%$ (number of samples was 6 ), respectively. These percentage values were much 


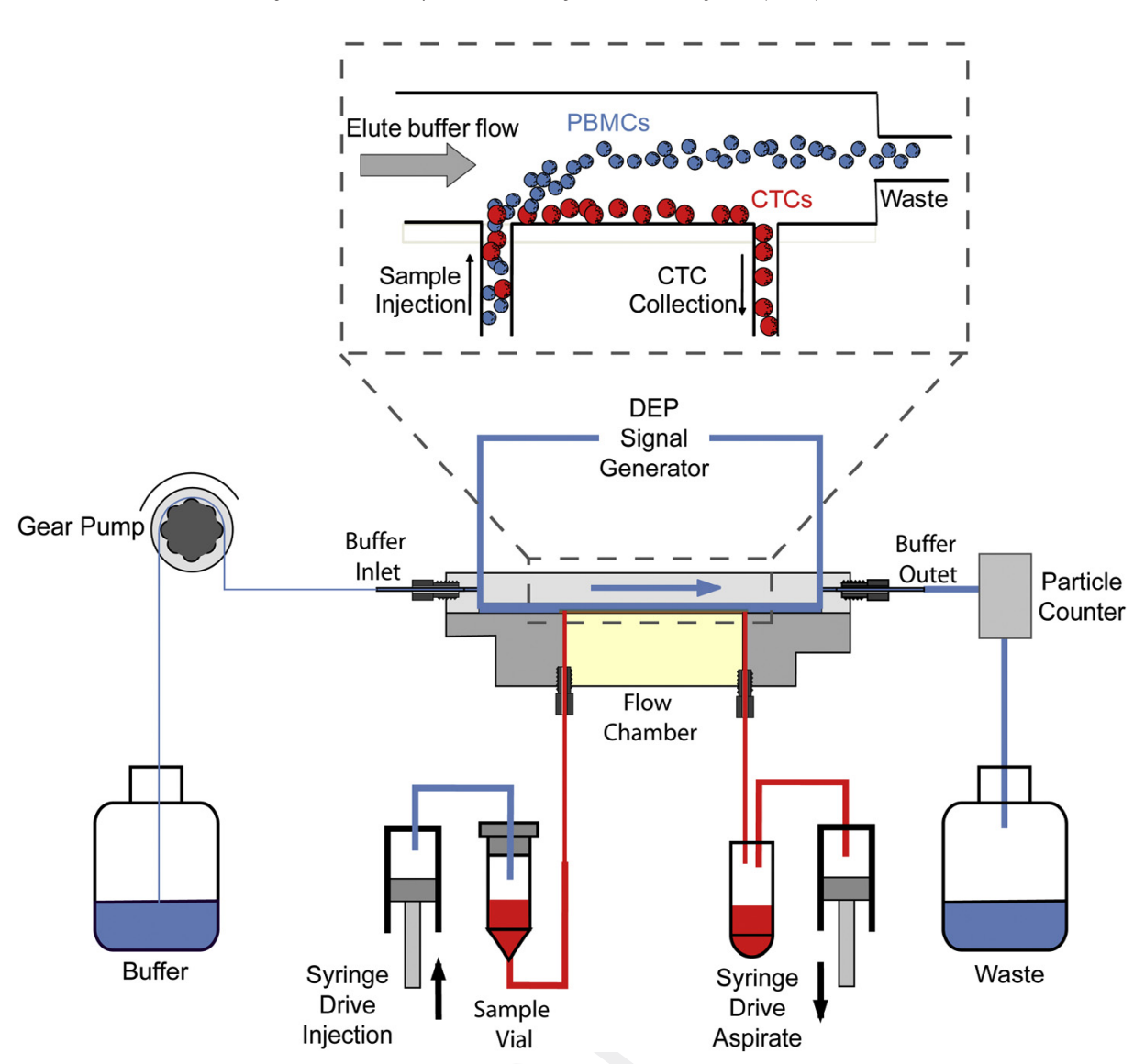

Fig. 4. A continuous flow dielectrophoresis (DEP) microfluidic cell-sorting device.

higher than in other reports using the DEP method [52] and the viability of the captured MDA-MB-231 cancer cells was greater than $97.1 \%$.

Lee and co-workers developed a high-purity CTC-isolation technique based on optically-induced DEP (ODEP) [62], which featured a sandwich structure with top and bottom layers made of indium-tin-oxide (ITO) glass, and the bottom layer had a photoconductive coating (hydrogenated amorphous silicon). Between these layers, there was a liquid layer containing the sample. An alternatingcurrent $(A C)$ voltage was first applied in the ODEP system to produce a uniform electric field. After optical illumination, electron-hole pairs were generated in the photoconductive layer, causing significant increase in the conductivity of the bottom layer. This phenomenon allowed the applied voltage to drop across the liquid layer, producing a locally non-uniform electric field at the illuminated regions [63]. With this method, the recovery rate was $76-83 \%$ for PC-3 cells, and $61-68 \%$ for OEC-M1 cells with $\sim 95 \%$ viability [62]. The viability of the captured CTCs also depended on the applied voltage and the frequency of the DEP field, so DEP operation conditions needed to be carefully optimized to avoid collection of non-viable cells $[64,65]$. A low-intensity, intermediate-frequency alternating electric field (100-300 kHz) might have an inhibitory effect on the growth rate of a variety of human-tumor cell lines [66].

\section{Magnetic trapping}

Magnetic-trapping techniques utilize magnetic particles with magnetic-field manipulation. Magnetic particles are typically sized in the range of several nanometers to a couple of micrometers and they have a magnetic core and a non-magnetic coating that can be chemically modified $[15,67]$. In microfluidic systems, both permanent magnets and/or electromagnets can be used. Passive magnetic [68] elements or electromagnets $[69,70]$ are usually assembled in the wall of the microchannels to trap the magnetic particles at the surface of the microchannel.

Effective capture of MCF-7 breast-cancer cells in a microfluidic chip with self-assembled protein-coated magnetic beads was demonstrated by Sivagnanam et al. [71]. The beads were patterned in situ inside a sealed microfluidic channel by magnetic-field-assisted electrostatic self-assembly and grafted by 5D10 monoclonal antibodies. The capture efficiency of the system for MCF-7 cells was $85 \pm 10 \%$.

Superparamagnetic beads were also utilized for magnetic cell capture. In the so-called Ephesia system, these beads self-assembled in a microfluidic channel into an array of magnetic traps prepared by microcontact printing. This approach combined the advantages of microfluidic cell sorting, notably the application of a wellcontrolled, flow-activated interaction between cells and beads, and immunomagnetic sorting. The superparamagnetic beads were antibody (anti-EpCAM)-coated for CTC capture [72]. Under a moderate external magnetic field, dipole-dipole interactions between the magnetic bead particles formed chains aligned along the electric field direction forming nanopillar-like structures [73]. The principle of this technology is depicted in Fig. 5. Cell-capture yield reached 94\% with this approach.

Another interesting approach is the utilization of magnetophoretic mobility [i.e. the motion of particles in a viscous medium induced by a magnetic field on a particle or cell of magnetic or magnetizable material (e.g. magnetically-labeled cells)] [74]. Murthy and coworkers developed a continuous-flow, magnetophoretic, microfluidic, 


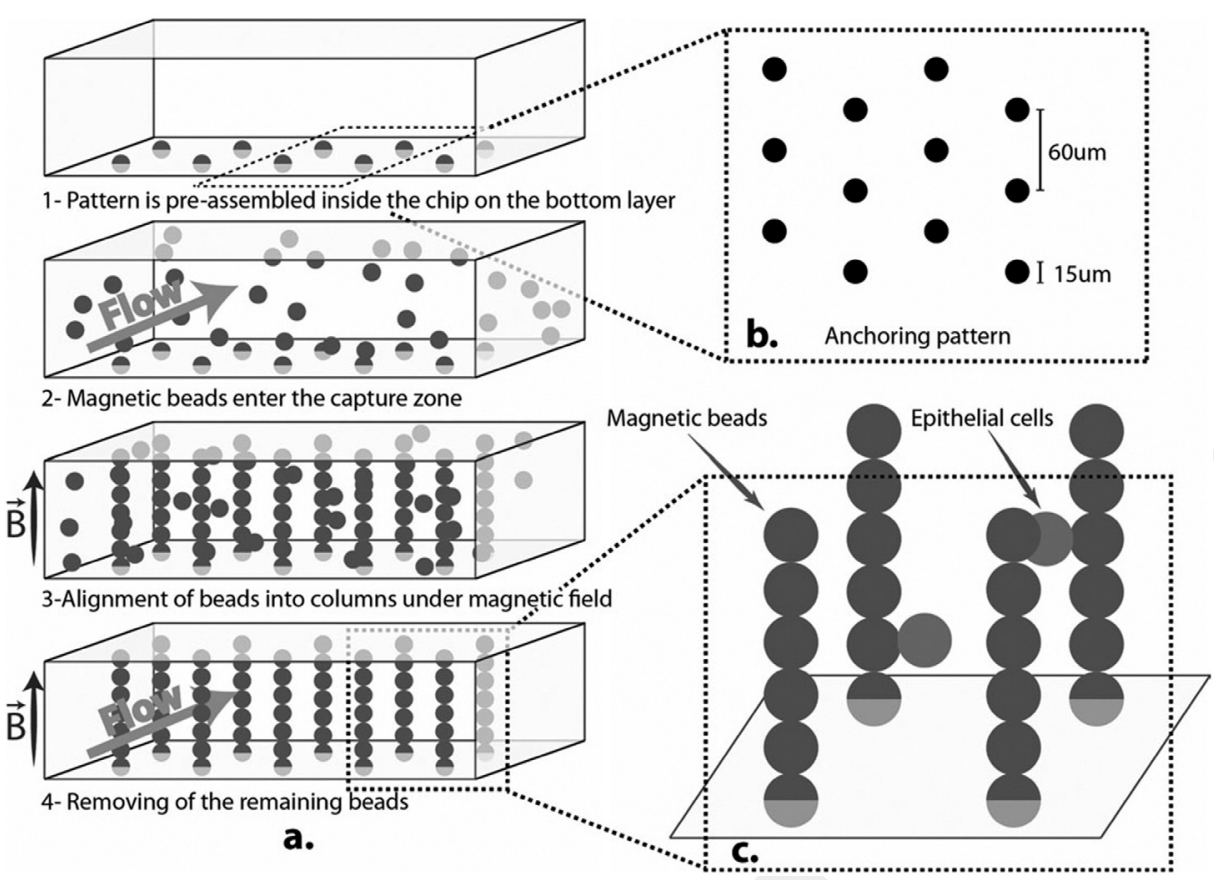

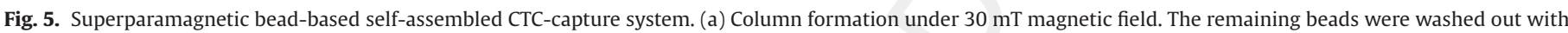

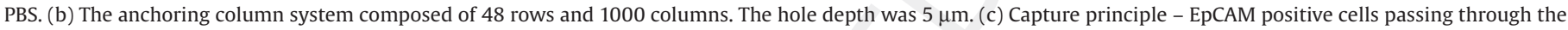
column system are caught by antibody/antigen interactions. (O)

rare-cell-capture device with the principle of applying a laminar flow in the $y$-direction and a magnetic field in the $x$-direction, so the magnetically-tagged cells moved from the outside sample streams to the center collection stream (Fig. 6) [75]. To test the capability of the set-up, $50 \mathrm{MCF}-7$ cells were spiked into $1 \mathrm{fmL}$ blood from healthy volunteers. The blood samples were then mixed with Dynal MyOne anti-EpCAM-functionalized magnetic beads and applied. The capture efficiency was greater than $85 \%$ with purity over $90 \%$ [75].

An interesting approach with a negative CTC-enrichment technique was also developed utilizing the combination of viscous flow and magnetic force to facilitate the recovery of unlabeled cells (CTCs) from whole-blood samples obtained from cancer patients [76,77]. The method was based on removal of erythrocytes by lysis followed by the magnetic separation of immunomagnetically-labeled (with CD45 antibody) leukocytes. After the magnetic separation, the remaining cells were considered mostly CTCs.

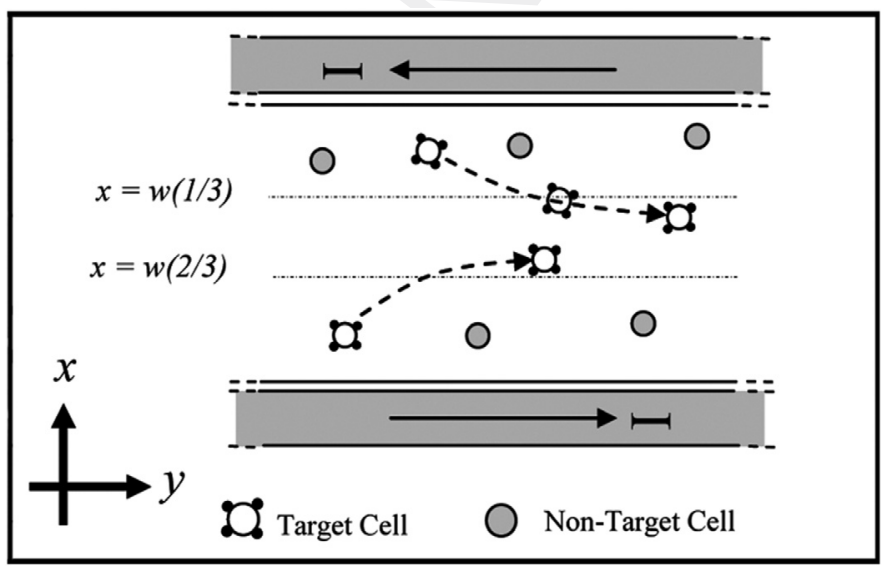

Fig. 6. Magnetophoretic cell-separation principle.

\section{Conclusions and future prospects}

Recent advances in nanotechnology and microfluidics have made possible the design and the implementation of highly-reliable CTCcapture platforms with excellent yield and selectivity for the most common types of cancer cell. Capture systems with high throughput, rapid processing times and minimal false-positive rates are highly desirable in the medical and diagnostic fields to monitor progress during cancer therapy and, more importantly, for early cancer detection. Molecular profiling of CTCs can also help to identify new targets and so can aid development of novel therapeutic agents. For wider acceptance, the capabilities of CTC targeting microfluidic detection and capture devices should be demonstrated on a large variety of cancer types. In addition, for proper regulatory approvals, the analytical validity of the CTC assay should be rigorously tested based on Clinical Laboratory Improvement Amendments (CLIA) or Good Laboratory Practice (GLP) standards.

We expect that, in the near future, once such microfluidic CTC detection and capture devices are approved by the regulatory agencies, they will quickly find their way for routine use in personalized therapeutics for cancer patients.

\section{Acknowledgment}

The authors gratefully acknowledge the support of the Momentum (Lendulet) grant \# 97101 of the Hungarian Academy of Sciences (MTA-PE Translational Glycomics) and TAMOP-4.2.2.A-11/1/ KONC-2012-0072.

\section{References}

[1] C. Alix-Panabieres, H. Schwarzenbach, K. Pantel, Circulating tumor cells and circulating tumor DNA, Annu. Rev. Med. 63 (2012) 199-215.

[2] K. Pantel, C. Alix-Panabieres, S. Riethdorf, Cancer micrometastases, Nat. Rev. Clin. Oncol. 6 (2009) 339-351.

[3] B.T. Greene, A.D. Hughes, M.R. King, Circulating tumor cells: the substrate of personalized medicine?, Front. Oncol. 2 (2012) 69. 
[4] B. Faltas, Cornering metastases: therapeutic targeting of circulating tumor cells and stem cells, Front. Oncol. 2 (2012) 68.

[5] M. Yu, S. Stott, M. Toner, S. Maheswaran, D.A. Haber, Circulating tumor cells: approaches to isolation and characterization, J. Cell Biol. 192 (2011) 373-382.

[6] K. Pantel, R.H. Brakenhoff, Dissecting the metastatic cascade, Nat. Rev. Cancer 4 (2004) 448-456.

[7] M. Wendel, L. Bazhenova, R. Boshuizen, A. Kolatkar, M. Honnatti, E.H. Cho, et al., Fluid biopsy for circulating tumor cell identification in patients with early-and late-stage non-small cell lung cancer: a glimpse into lung cancer biology, Phys. Biol. 9 (2012) 016005.

[8] D. Marrinucci, K. Bethel, A. Kolatkar, M.S. Luttgen, M. Malchiodi, F. Baehring, et al., Fluid biopsy in patients with metastatic prostate, pancreatic and breast cancers, Phys. Biol. 9 (2012) 016003.

[9] R. Boshuizen, P. Kuhn, M. van den Heuvel, Circulating tumor cells in non-small cell lung carcinoma, J. Thorac. Dis. 4 (2012) 456-458.

[10] US Food and Drug Administration: Draft guidance for industry and food and drug administration staff: in vitro companion diagnostic devices DRAFT GUIDANCE. (2011). http://www.fda.gov/downloads/MedicalDevices/ DeviceRegulationandGuidance/GuidanceDocuments/UCM262327.pdf.

[11] US Food and Drug Administration: FDA's clearance to CellSearch ${ }^{\mathrm{TM}}$ epithelial cell kit/CellSpotter ${ }^{\mathrm{TM}}$ analyzer. (2004). http://www.fda.gov/MedicalDevices/ ProductsandMedicalProcedures/DeviceApprovalsandClearances/RecentlyApprovedDevices/ucm081239.htm.

[12] M. Cristofanilli, L.W.M.M. Terstappen, D.F. Hayes, Circulating epithelial cells in breast cancer - reply, N. Engl. J. Med. 351 (2004) 2453-2454

[13] R.Y. Chein, Y.C. Yang, Y.S. Lin, Estimation of Joule heating effect on temperature and pressure distribution in electrokinetic-driven microchannel flows, Electrophoresis 27 (2006) 640-649.

[14] R.N. Zare, S. Kim, Microfluidics platforms for single-cell analysis, Annu. Rev. Biomed. Eng. 12 (2010) 187-201.

[15] J. Nilsson, M. Evander, B. Hammarstrom, T. Laurell, Review of cell and particle trapping in microfluidic systems, Anal. Chim. Acta 649 (2009) 141-157.

[16] A.D. Hughes, M.R. King, Nanobiotechnology for the capture and manipulation of circulating tumor cells, Wiley Interdiscip. Rev. Nanomed. Nanobiotechnol. 4 (2012) 291-309.

[17] K. Pantel, C. Alix-Panabieres, Circulating tumour cells in cancer patients: challenges and perspectives, Trends Mol. Med. 16 (2010) 398-406.

[18] E.A. Punnoose, S.K. Atwal, J.M. Spoerke, H. Savage, A. Pandita, R.F. Yeh, et al., Molecular biomarker analyses using circulating tumor cells, PLoS ONE 5 (2010) e12517.

[19] T.M. Gorges, K. Pantel, Circulating tumor cells as therapy-related biomarkers in cancer patients, Cancer Immunol. Immunother. 62 (2013) 931-939.

[20] P. Wulfing, J. Borchard, H. Buerger, S. Heidl, K.S. Zanker, L. Kiesel, et al. HER2-positive circulating tumor cells indicate poor clinical outcome in stage I to III breast cancer patients, Clin. Cancer Res. 12 (2006) 1715-1720.

[21] W. Onstenk, J.W. Gratama, J.A. Foekens, S. Sleijfer, Towards a personalized breast cancer treatment approach guided by circulating tumor cell (CTC) characteristics, Cancer Treat. Rev. 39 (2013) 691-700.

[22] S. Maheswaran, L.V. Sequist, S. Nagrath, L. Ulkus, B. Brannigan, C.V. Collura, et al., Detection of mutations in EGFR in circulating lung-cancer cells, N. Engl. J. Med. 359 (2008) 366-377.

[23] Z.H. Wu, K. Hjort, Microfluidic hydrodynamic cell separation: a review, Micro Nanosyst. 1 (2009) 181-192.

[24] Y. Sai, M. Yamada, M. Yasuda, M. Seki, Continuous separation of particles using a microfluidic device equipped with flow rate control valves, J. Chromatogr. A 1127 (2006) 214-220.

[25] Z.G. Wu, A.Q. Liu, K. Hjort, Microfluidic continuous particle/cell separation via electroosmotic-flow-tuned hydrodynamic spreading, J. Micromech. Microeng. 17 (2007) 1992-1999.

[26] D.W. Inglis, Efficient microfluidic particle separation arrays, Appl. Phys. Lett. 94 (2009) 013510

[27] M. Yamada, M. Nakashima, M. Seki, Pinched flow fractionation: continuous size separation of particles utilizing a laminar flow profile in a pinched microchannel, Anal. Chem. 76 (2004) 5465-5471.

[28] D. Di Carlo, Inertial microfluidics, Lab Chip 9 (2009) 3038-3046.

[29] S.S. Shevkoplyas, T. Yoshida, L.L. Munn, M.W. Bitensky, Biomimetic autoseparation of leukocytes from whole blood in a microfluidic device, Anal. Chem. 77 (2005) 933-937.

[30] M.L. Turgeon, Clinical Hematology: Theory and Procedures, Fourth ed., Lippincott Williams \& Wilkins, Baltimore, Philadelphia, 2005, p. 100.

[31] G. Vona, A. Sabile, M. Louha, V. Sitruk, S. Romana, K. Schutze, et al., Isolation by size of epithelial tumor cells - a new method for the immunomorphological and molecular characterization of circulating tumor cells, Am. J. Pathol. 156 (2000) 57-63.

[32] H.M. Shapiro, E.R. Schildkraut, R. Curbelo, C.W. Laird, R.B. Turner, T. Hirschfeld, Combined blood-cell counting and classification with fluorochrome stains and flow instrumentation, J. Histochem. Cytochem. 24 (1976) 396-411.

[33] S. Zheng, H. Lin, J.Q. Liu, M. Balic, R. Datar, R.J. Cote, et al., Membrane microfilter device for selective capture, electrolysis and genomic analysis of human circulating tumor cells, J. Chromatogr. A 1162 (2007) 154-161.

[34] S.Y. Zheng, H.K. Lin, B. Lu, A. Williams, R. Datar, R.J. Cote, et al., 3D microfilter device for viable circulating tumor cell (CTC) enrichment from blood, Biomed. Microdevices 13 (2011) 203-213.

[35] M. Hosokawa, T. Hayata, Y. Fukuda, A. Arakaki, T. Yoshino, T. Tanaka, et al., Size-selective microcavity array for rapid and efficient detection of circulating tumor cells, Anal. Chem. 82 (2010) 6629-6635.
[36] M. Hosokawa, T. Yoshikawa, R. Negishi, T. Yoshino, Y. Koh, H. Kenmotsu, et al., Microcavity array system for size-based enrichment of circulating tumor cells from the blood of patients with small-cell lung cancer, Anal. Chem. 85 (2013) 5692-5698.

[37] T.M. Geislinger, B. Eggart, S.B. Ller, L. Schmid, T. Franke, Separation of blood cells using hydrodynamic lift, Appl. Phys. Lett. 100 (2012) 183701.

[38] D. Di Carlo, D. Irimia, R.G. Tompkins, M. Toner, Continuous inertial focusing, ordering, and separation of particles in microchannels, Proc. Natl. Acad. Sci. U.S.A. 104 (2007) 18892-18897.

[39] A.A.S. Bhagat, S.S. Kuntaegowdanahalli, I. Papautsky, Continuous particle separation in spiral microchannels using dean flows and differential migration, Lab Chip 8 (2008) 1906-1914.

[40] A. Russom, A.K. Gupta, S. Nagrath, D. Di Carlo, J.F. Edd, M. Toner, Differential inertial focusing of particles in curved low-aspect-ratio microchannels, New J. Phys. 11 (2009) 075025.

[41] A.A.S.H. Bhagat, H.W. Hou, L.D. Li, C.T. Lim, J. Han, Dean Flow Field Fractionation (DFF) Isolation of Circulating Tumor Cells (CTCs) from Blood, Seattle, Washington, USA, 2011, pp. 524-526.

[42] J.S. Sun, C. Liu, M.M. Li, J.D. Wang, Y.L. Xianyu, G.Q. Hu, et al., Size-based hydrodynamic rare tumor cell separation in curved microfluidic channels, Biomicrofluidics 7 (2013) 011802.

[43] S.V. Litvinov, M.P. Velders, H.A.M. Bakker, G.J. Fleuren, S.O. Warnaar, Ep-cam - a human epithelial antigen is a homophilic cell-cell adhesion molecule, J. Cell Biol. 125 (1994) 437-446.

[44] S. Nagrath, L.V. Sequist, S. Maheswaran, D.W. Bell, D. Irimia, L. Ulkus, et al., Isolation of rare circulating tumour cells in cancer patients by microchip technology, Nature 450 (2007) 1235-1239.

[45] J.P. Gleghorn, E.D. Pratt, D. Denning, H. Liu, N.H. Bander, S.T. Tagawa, et al., Capture of circulating tumor cells from whole blood of prostate cancer patients using geometrically enhanced differential immunocapture (GEDI) and a prostate-specific antibody, Lab Chip 10 (2010) 27-29.

[46] S.T. Wang, H. Wang, J. Jiao, K.J. Chen, G.E. Owens, K.I. Kamei, et al., ThreeDimensional nanostructured substrates toward efficient capture of circulating tumor cells, Angew. Chem. Int. Ed. Engl. 48 (2009) 8970-8973.

[47] S. Iyer, R.M. Gaikwad, V. Subba-Rao, C.D. Woodworth, I. Sokolov, Atomic force microscopy detects differences in the surface brush of normal and cancerous cells, Nat. Nanotechnol. 4 (2009) 389-393.

[48] A.M. Shah, M. Yu, Z. Nakamura, J. Ciciliano, M. Ulman, K. Kotz, et al., Biopolymer system for cell recovery from microfluidic cell capture devices, Anal. Chem. 84 (2012) 3682-3688

[49] S.L. Stott, C.H. Hsu, D.I. Tsukrov, M. Yu, D.T. Miyamoto, B.A. Waltman, et al., Isolation of circulating tumor cells using a microvortex-generating herringbone-chip, Proc. Natl. Acad. Sci. U.S.A. 107 (2010) 1839218397.

[50] R. Pethig, Review article-dielectrophoresis: status of the theory, technology, and applications, Biomicrofluidics 4 (2010) 022811.

[51] K. Khoshmanesh, C. Zhang, F.J. Tovar-Lopez, S. Nahavandi, S. Baratchi, A. Mitchell, et al., Dielectrophoretic-activated cell sorter based on curved microelectrodes, Microfluid. Nanofluid. 9 (2010) 411-426.

[52] P.R.C. Gascoyne, J. Noshari, T.J. Anderson, F.F. Becker, Isolation of rare cells from cell mixtures by dielectrophoresis, Electrophoresis 30 (2009) 13881398.

[53] A.C. Sabuncu, J.A. Liu, S.J. Beebe, A. Beskok, Dielectrophoretic separation of mouse melanoma clones, Biomicrofluidics 4 (2010) 021101.

[54] H.S. Moon, K. Kwon, S.I. Kim, H. Han, J. Sohn, S. Lee, et al., Continuous separation of breast cancer cells from blood samples using multi-orifice flow fractionation (MOFF) and dielectrophoresis (DEP), Lab Chip 11 (2011) 1118-1125.

[55] P. Gascoyne, C. Mahidol, M. Ruchirawat, J. Satayavivad, P. Watcharasit, F.F. Becker, Microsample preparation by dielectrophoresis: isolation of malaria, Lab Chip 2 (2002) 70-75.

[56] Y. Huang, S. Joo, M. Duhon, M. Heller, B. Wallace, X. Xu, Dielectrophoretic cell separation and gene expression profiling on microelectronic chip arrays, Anal. Chem. 74 (2002) 3362-3371.

[57] R.S.W. Thomas, P.D. Mitchell, R.O.C. Oreffo, H. Morgan, Trapping single human osteoblast-like cells from a heterogeneous population using a dielectrophoretic microfluidic device, Biomicrofluidics 4 (2010) 022806.

[58] F. Yang, X.M. Yang, H. Jiang, P. Bulkhaults, P. Wood, W. Hrushesky, et al., Dielectrophoretic separation of colorectal cancer cells, Biomicrofluidics 4 (2010) 013204.

[59] H. Shafiee, J.L. Caldwell, M.B. Sano, R.V. Davalos, Contactless dielectrophoresis: a new technique for cell manipulation, Biomed. Microdevices 11 (2009) 997-1006.

[60] E.A. Henslee, M.B. Sano, A.D. Rojas, E.M. Schmelz, R.V. Davalos, Selective concentration of human cancer cells using contactless dielectrophoresis, Electrophoresis 32 (2011) 2523-2529.

[61] V. Gupta, I. Jafferji, M. Garza, V.O. Melnikova, D.K. Hasegawa, R. Pethig, et al., ApoStream (TM), a new dielectrophoretic device for antibody independent isolation and recovery of viable cancer cells from blood, Biomicrofluidics 6 (2012) 024133

[62] S.B. Huang, M.H. Wu, Y.H. Lin, C.H. Hsieh, C.L. Yang, H.C. Lin, et al., High-purity and label-free isolation of circulating tumor cells (CTCs) in a microfluidics platform by using optically-induced-dielectrophoretic (ODEP) force, Lab Chip 13 (2013) 1371-1383.

[63] W.Y. Lin, Y.H. Lin, G.B. Lee, Separation of micro-particles utilizing spatial difference of optically induced dielectrophoretic forces, Microfluid. Nanofluid. 8 (2010) 217-229. 
[64] L. Yang, P.P. Banada, A.K. Bhunia, R. Bashir, Effects of dielectrophoresis on growth, viability and immuno-reactivity of listeria monocytogenes, J. Biol. Eng. 2 (2008) 6.

[65] A. Alazzam, I. Stiharu, R. Bhat, A.N. Meguerditchian, Interdigitated comb-like electrodes for continuous separation of malignant cells from blood using dielectrophoresis, Electrophoresis 32 (2011) 1327-1336.

[66] E.D. Kirson, Z. Gurvich, R. Schneiderman, E. Dekel, A. Itzhaki, Y. Wasserman, et al., Disruption of cancer cell replication by alternating electric fields, Cancer Res. 64 (2004) 3288-3295.

[67] M.A.M. Gijs, Magnetic bead handling on-chip: new opportunities for analytical applications, Microfluid. Nanofluid. 1 (2004) 22-40.

[68] K. Smistrup, T. Lund-Olesen, M.F. Hansen, P.T. Tang, Microfluidic magnetic separator using an array of soft magnetic elements, J. Appl. Phys. 99 (2006) 08 p102.

[69] J.W. Choi, T.M. Liakopoulos, C.H. Ahn, An on-chip magnetic bead separator using spiral electromagnets with semi-encapsulated permalloy, Biosens. Bioelectron. 16 (2001) 409-416.

[70] Q. Ramadan, V. Samper, D. Poenar, C. Yu, On-chip micro-electromagnets for magnetic-based bio-molecules separation, J. Magn. Magn. Mater. 281 (2004) $150-172$.

[71] V.Sivagnanam, B. Song, C. Vandevyver, J.C.G. Bunzli, M.A.M. Gijs, Selective breast cancer cell capture, culture, and immunocytochemical analysis using self- assembled magnetic bead patterns in a microfluidic chip, Langmuir 26 (2010) 6091-6096.

[72] A.E. Saliba, L. Saias, E. Psychari, N. Minc, D. Simon, F.C. Bidard, et al., Microfluidic sorting and multimodal typing of cancer cells in self-assembled magnetic arrays, Proc. Natl. Acad. Sci. U.S.A. 107 (2010) 14524-14529.

[73] D. Horak, Z. Svobodova, J. Autebert, B. Coudert, Z. Plichta, K. Kralovec, et al., Albumin-coated monodisperse magnetic poly(glycidyl methacrylate) microspheres with immobilized antibodies: application to the capture of epithelial cancer cells, J. Biomed. Mater. Res. A 101A (2013) 23-32.

[74] S. Winoto-Morbach, V. Tchikov, W. Muller-Ruchholtz, Magnetophoresis: I. Detection of magnetically labeled cells, J. Clin. Lab. Anal. 8 (1994) 400406.

[75] B.D. Plouffe, M. Mahalanabis, L.H. Lewis, C.M. Klapperich, S.K. Murthy, Clinically relevant microfluidic magnetophoretic isolation of rare-cell populations for diagnostic and therapeutic monitoring applications, Anal. Chem. 84 (2012) 1336-1344.

[76] M. Zborowski, J.J. Chamers, Rare cell separation and analysis by magnetic sorting, Anal. Chem. 83 (2011) 8050-8056.

[77] L.Y. Yang, J.C. Lang, P. Balasubramanian, K.R. Jatana, D. Schuller, A. Agrawal, et al., Optimization of an enrichment process for circulating tumor cells from the blood of head and neck cancer patients through depletion of normal cells, Biotechnol. Bioeng. 102 (2009) 521-534. 